Calderón, A., Ruiz, M., \& O’Connor, R. V. (2017). ProDecAdmin: A Game Scenario Design Tool for Software Project Management Training. In J. Stolfa, S. Stolfa, R. V. O'Connor, \& R. Messnarz (Eds.), Systems, Software and Services Process Improvement: 24th European Conference, EuroSPI 2017, Ostrava, Czech Republic, September 6-8, 2017, Proceedings (pp. 241-248). Cham: Springer International Publishing. https://doi.org/10.1007/978-3-319-642185_19

\title{
ProDecAdmin: A Game Scenario Design Tool for Software Project Management Training
}

\author{
Alejandro Calderón ${ }^{1}$, Mercedes Ruiz ${ }^{1}$, Rory V. O'Connor ${ }^{2}$ \\ ${ }^{1}$ University of Cádiz, Cádiz, Spain. \\ \{alejandro.calderon, mercedes.ruiz\}@uca.es \\ ${ }^{2}$ Dublin City University, Dublin, Ireland. \\ rory.oconnoredcu.ie
}

\begin{abstract}
Teaching Software Project Management (SPM) for Information Technology (IT) learners is a relevance issue. The necessity of teaching SPM in a highly practical way moves trainers towards the use of new methods and techniques such as simulations, serious games or gamification strategies. The majority of the existing serious games for SPM do not offer flexibility, in terms of the ability to provide and dynamically change game scenarios during the life of the serious game, and not allow assessing learners' new skill automatically. In this paper, we introduce the administration tool of the serious game ProDec, that allow trainers to design the game scenarios of the game trying to overcome the lacks found in the scope of serious games for SPM.
\end{abstract}

Keywords: Gamification, Serious Game, Game Scenario Design, Software Project Management, Education

\section{Introduction}

Teaching SPM for IT learners is a topical issue that has always been supported by organizations such as the Association for Computing Machinery (ACM) and IEEEComputer Society (ACM/IEEE-CS Joint Task Force on Computing Curricula, 2013). These organizations have not only highlighted the importance of this topic, but they have also emphasized the need of teaching SPM in a highly practical way, where learners can put into practice their knowledge in real-life scenarios. This necessity moves trainers towards the use of new methods and techniques that allow teaching in a highly practical way, promoting active learning and increasing the motivation and the engagement of learners in the learning-teaching process of SPM (Kosa, Yilmaz, O'Connor, \& Clarke, 2016; Martí-Parreño, Méndez-Ibáñez, \& Alonso-Arroyo, 2016).

Gamification is "the use of game elements and game design techniques in nongame contexts" (Werbach \& Hunter, 2012). It has become "an increasingly popular 
approach to increasing end-user engagement in many contexts, including employee productivity, marketing, citizens awareness, and education" (Sheth, Bell, \& Kaiser, 2012). The design and use of gamification strategies are one of the new methods using to improve user's engagement, motivation, and performance within the learning-teaching process and to provide a more interesting education in software engineering (Pedreira, García, Brisaboa, \& Piattini, 2015). Once of the common practice in the road to apply gamification in education is the use of games as a component of the gamification strategy (Yildirim, 2017). The use of games and simulation-based experiences allows learners to experiment, learn from their own mistakes and acquire experience with real-life scenarios within a free-risk environment. These kinds of games, designed with a different purpose than only entertainment, are called serious games (Abt, 2002; Zyda, 2005).

Regarding the scope of serious games for training SPM, there are a limited number of games available (Caulfield, Xia, Veal, \& Maj, 2011; Calderón \& Ruiz, 2015; Petri \& von Wangenheim, 2017), with well-known examples being: SimSoft (Caulfield, Veal, \& Maj, 2011), SimSE (Navarro \& Hoek, 2004), X-MED (Von Wangenheim, Thiry, \& Kochanski, 2009), DELIVER! (Von Wangenheim, Savi, \& Borgatto, 2012). The analysis of the current proposals of serious games for teaching SPM allows shows their main weaknesses in this scope are that: (a) they focus on learning specific techniques of project management or specific stages of the project's lifecycle; (b) they do not usually reach all levels of Bloom's taxonomy; (c) they do not allow assessing learner's new skills automatically; and (d) their flexibility in terms of game scenarios is very limited (Calderón \& Ruiz, Bringing real-life practice in software project management through a simulation-based serious game, 2014).

Regarding the lack of the serious games for training SPM, in this paper, we introduce the administration tool of ProDec, a simulation-based serious game for SPM. This tool tries to overcome the main weaknesses found in this scope by allowing trainers to design the game scenarios of the serious game. The structure of the paper is as follows: Section 2 shows the background of this work. Section 3 introduces the administration tool of ProDec for game scenarios design. Finally, Section 4 summarizes the paper and presents our conclusions and future work.

\section{Background}

In previous works, we have introduced ProDec (Calderón \& Ruiz, ProDec: a serious game for software project management training, 2013), a simulation-based serious game to teach, motivate and assess learners in the learning-teaching process of SPM concepts and practices, and we have analyzed different features about their capability to be integrated into the learning process (Calderón \& Ruiz, Bringing real-life practice in software project management through a simulation-based serious game, 2014) or their ability to cover some software processes of international standards (Calderón \& Ruiz, Coverage of ISO/IEC 12207 Software Lifecycle Process by a Simulation-Based Serious Game, 2016). 
Among these features we can find that ProDec's main goal is to place learners in a virtual environment where the can manage software projects and practice their knowledge with real-life scenarios in a risk-free environment. For this, the serious game provides two different modes of play, called "Full Play" and "Quick Play". In a "Full Play", learners create their own project plans from scratch and then, they execute the simulation of their own project scenario. On the other hand, in a "Quick Play", learners play with a project scenario created by trainers. In both modes of play, the main goal is to successful manage a project. This involves ending the project

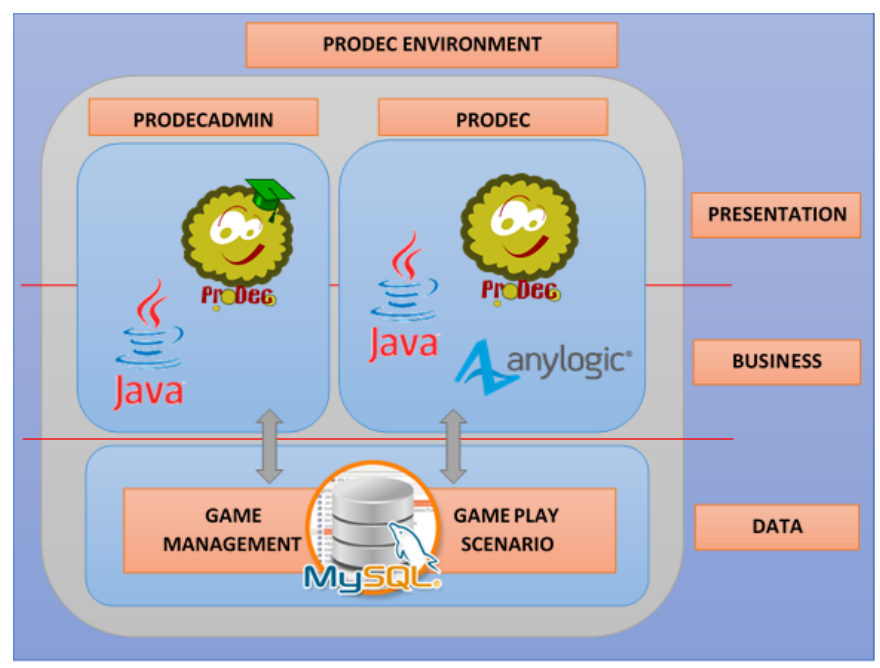

Figure 1. ProDec's architecture.

within the time and costs constraints established on the project plan of the scenario.

In order to allow these features, ProDec is part of a learning-teaching environment that follows a three-layer architecture and has been developed using Java, Anylogic and MySQL technologies. Two software tools developed using Java and the simulation model deal with the presentation and business layer and two databases managed by MySQL deal with the data layer of the architecture. As we can see in Figure 1, the ProDec environment is composed of the following applications:

a) ProDec is the software application (simulation-based serious game) used by the players. This application is composed of three main stages that allow players to engage in the game and put in practices their knowledge about SPM. The three main stages are described as follow:

- An initial stage, called Onset, where players chose the mode of play and create or select the project scenario that they want to play it. At the end of this stage, the game automatically generates the source file of the discrete-event simulation model required to simulate the project plan of the scenario. 
- A simulation stage, called Execution, where players execute the simulation of the project scenario and monitor it in order to cover the goal of the game.

- A final stage, called End, which finishes the game scenario and performs the players' assessment by applying the criteria set by the trainers for the scenario that has been played.

b) ProDecAdmin is the software application that allows trainers to manage all the information required by ProDec. The trainers use this application to design the different game scenarios for the "Quick Play" mode and to set the rubrics for players' assessment.

Taking into account the architecture of ProDec environment, in the following sections, we introduce ProDecAdmin, the administration tool of ProDec for trainers that allows them to design game scenarios for teaching SPM according to their learning goals.

\section{ProDecAdmin}

ProDecAdmin is an application to be used by trainers within the ProDec environment with the goal that they can design all the needed elements for the correct operation of ProDec and for assessing of the players' performance through the ProDec scenarios (see Figure 2). In order to cover the functionalities mentioned above, the tool allows trainers to perform the following main activities: (a) creating all the needed elements to design project plans that will be used by ProDec; (b) establishing the criteria to assess players' performance during the use of ProDec; and (c) creating game scenarios for the "Quick Play" mode of ProDec.

In the following subsections, we introduce the main features of ProDecAdmin and the elements involve in the process of designing a game scenario for the "Quick Play" mode of ProDec.

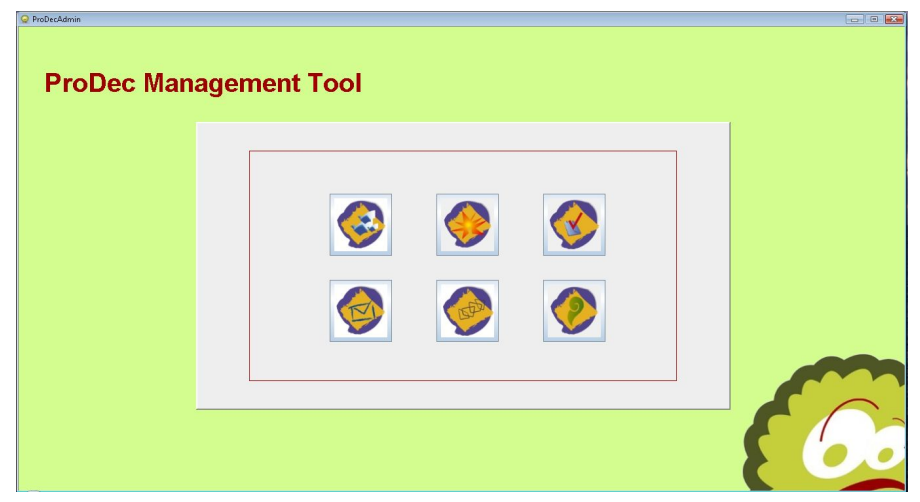

Figure 2. ProDecAdmin's main screen. 


\subsection{Designing Project Plans}

A project plan is the main element of a game scenario in ProDec. As we have commented previously, a project plan can be created by players through the "Full Play" mode of ProDec or can be created by trainers to design a scenario for the "Quick Play" mode of ProDec.

The administration tool, ProDecAdmin, allows trainers to create these project plans as a part of the process to design a game scenario. For this, the tool provides trainers three main functionalities: tasks, risks and project management. The functionalities of tasks and risk management allows trainers to design general project tasks and project risks that can be included in a project during its design.

Furthermore, the project management tool allows trainers to manage and design project plans. As we can see in Figure 3, to design a project plan, trainers have to follow a five-stage process that guides them from the definition of the project context to the definition of the risks that can be occurred during the project execution. These five stages are defined as following:

- Project Information (PI). In this stage, trainers provide the general information of the project such as its scope, objective, specifications, etc. and the specific information of the project that is needed to start the size estimation stage. Through this stage, trainers define the context of the project scenario and the complexity of the project in terms of the project requirements and duration. 
- Size Estimation (SE). In this stage, trainers perform the size estimation of the project. This stage allows trainers to estimate the effort in term of human resources and costs of the project.

- Project Team Definition (PTD). In this stage, trainers design and define the human resources of the project and establish the composition of the work team. This stage also allows trainers to design the features in terms of experience and personality traits of each human resource involves in the project work team.

- Tasks Definition (TD). In this stage, trainers define the project tasks, estimate the duration of each of them, allocate the human resources for each task and create a schedule of the project tasks taking into account the estimated start and completion dates, the assigned human resources and the dependency of the tasks. Through this stage, trainers can increase or reduce the complexity of the game scenario depending on the number of tasks, the human resources involve and the different

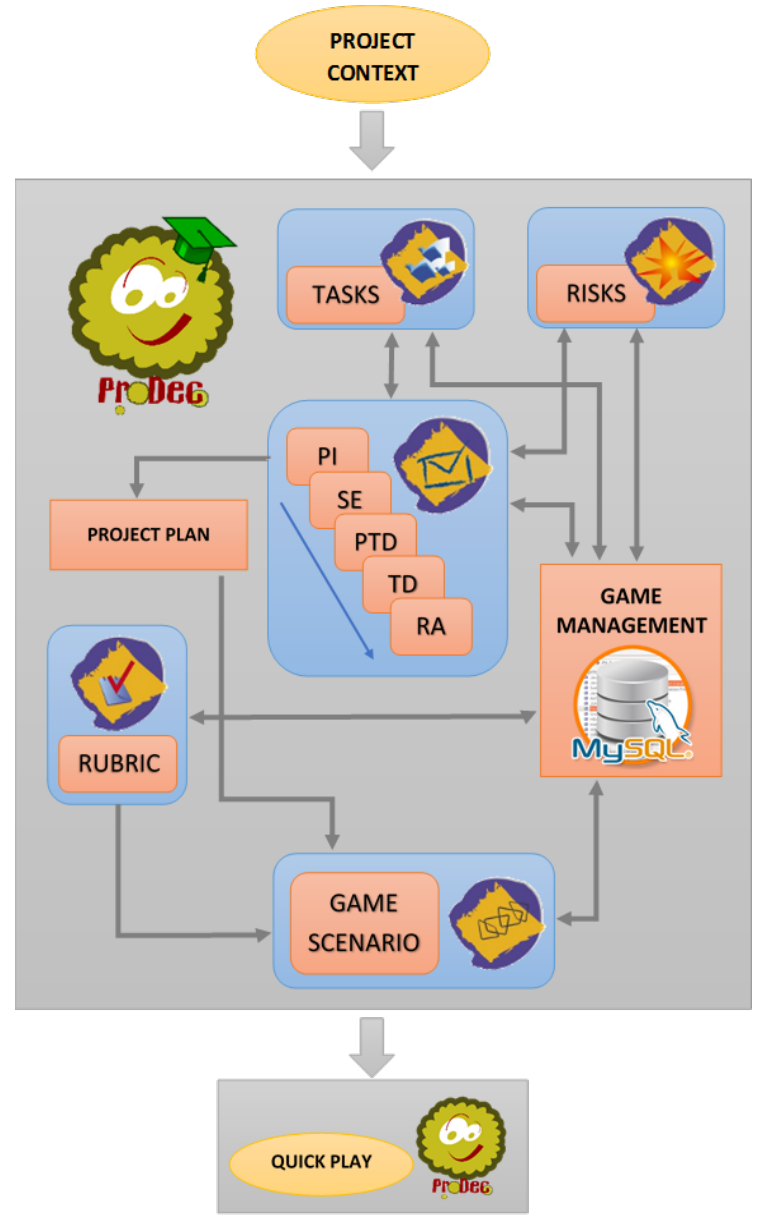

Figure 3. Game scenario design process. 
combination of teams and tasks dependency.

- Risks Analysis (RA). In this stage, trainers define the risks that can occur during the project execution. This means that trainers define the risks that can produce changes in a game scenario during its execution.

At the end of this process, trainers have evolved a project plan that can be used in a game scenario, with complexity dependent upon the different features related to the tasks, risks and human resources features involve in the project. Moreover, the different elements involved in a project plan that trainers have to design, allows them to create project plans focusing on specific learning goals such as the influence of synergy, size or experience of the human resource in the productivity of a work team, the important of defining a good schedule of project tasks, etc.

\subsection{Designing Players Assessment}

The players' assessment process involve elements from several sources within ProDec environment such as the initial estimates, the project monitoring data and the kind of decisions that the players made during the play of a scenario. In this process, trainers need to define the rubrics that will be used to assess the players' performance during a game scenario. ProDecAdmin allows trainers to design these rubrics.

In the game, a rubric is composed of a set of sections. At the same time, each section is composed of a set of assessment criteria. An assessment criterion links the information recorded in the rubric with the information recorded during the game. By using a labelling system, the labels describing the skills of an assessment criterion are matched with the records of the game that contain the information needed to assess such criterion. Then, the design of a rubric involves the definition of each section and the selection of the labels that are going to be assessed by the criteria.

\subsection{Designing Game Scenarios}

Once trainers have designed a project plan and an assessment rubric, the last step in a game scenario design is to link the project scenario with the assessment rubric that trainers want to use in its evaluation process. Using ProDecAdmin, trainers are able to create new game scenarios for the "Quick Play" mode of ProDec by connecting a defined rubric with a designed project. As a result, players can select a new game scenario when they play a Quick Play with ProDec.

\section{Discussion and Further Works}

Regarding the relevance of teaching SPM in a highly practical way, there is no doubt that the use of gamification, simulation and serious games are good methods and techniques to bring real-life scenarios to the learning-teaching process and turn theoretical lectures in a more practical environment. In this paper, taking into account our previous works, we have identified the main lacks found in the scope of serious games for SPM and we have commented the main features of ProDec, with the goal 
to introduce ProDecAdmin, the administration tool of ProDec that provides trainers with an environment for designing game scenarios according to their learning goals.

Flexibility, defined as the ability of a serious game to provide game scenarios that can be changed dynamically during the game, is an important feature to take into account in a serious game for SPM. In a game for teaching SPM, if game scenarios are static, learners will always experience the same activities. This maybe uninteresting for learners and they may quickly lose interest. At the other side, if game scenarios change dynamically and on a real-time basis, learners can face different challenges and apply their knowledge in different situations. In addition, the lifecycle of the SG as a learning resource within a course can be longer, as trainers can set up the scenarios repetitively.

SPM is a subject where learners need to take into account an extensive amount of information during the whole project lifecycle and they need to analyze the information of the project and make decisions continuously. Thus, a serious game that allows automatic assessment helps a) trainers to analyze and evaluate all the actions performed by learners during the game scenario and b) learners to get instant feedback on their performance, helping them, at the same time, to improve their knowledge during the game scenario.

The features of ProDecAdmin provide a high degree of flexibility, thus trainers are able to design any game scenario for ProDec that they want in order to cover their learning goals. Moreover, it allows trainers to design the assessment criteria to evaluate the users' performance, knowledge acquisition and progression with the game, which will be used by the ProDec in order to provide an automatically assessment report of the game scenario played. Therefore, we can conclude that ProDecAdmin helps to overcome the lacks of flexibility and automatic assessment identified in the scope of serious games for SPM.

Finally, from our point of view, we believe that simulation tools, serious games and gamification strategies are needed to prepare new practitioners for their professional life. For that reason, we go on working in this scope in order to improve the features of ProDecAdmin and ProDec. Moreover, we are evaluating the benefits of integrating our game with social networks and designing a gamification strategy that integrates the use of ProDec, in order to enrich the learning-teaching process and allow learners to acquire the concepts and practices of SPM in a practical environment where they can experiment with real-life scenarios during their studies.

\section{Acknowledgements}

This work has been partially supported by the Spanish Ministry of Science and Technology with AEI/FEDER/UE funds (grants TIN2013-46928-C3-2-R and TIN2016-76956-C3-3-R) and the Andalusian Plan for Research, Development and Innovation (grant TIC-195).

ReferencesAbt, C. (2002). Serious Games. Lanhan, MD: University Press of America. 
ACM/IEEE-CS Joint Task Force on Computing Curricula. (2013). Computer Science Curricula 2013. ACM Press and IEEE Computer Society Press.

Calderón, A., \& Ruiz, M. (2015). A systematic literature review on serious games evaluation: An application to software project management. Computers \& Education , 87, 396-422.

Calderón, A., \& Ruiz, M. (2014). Bringing real-life practice in software project management through a simulation-based serious game. Proceedings of the 6th International Conference on Computer Supported Education. Barcelona, Spain.

Calderón, A., \& Ruiz, M. (2016). Coverage of ISO/IEC 12207 Software Lifecycle Process by a Simulation-Based Serious Game. Proceedings of SPICE, (pp. 59-70). Dublin, Ireland.

Calderón, A., \& Ruiz, M. (2013). ProDec: a serious game for software project management training. Proceedings of the 8th ICSEA. Venice, Italy.

Caulfield, C., Veal, D., \& Maj, S. (2011). Teaching Software Engineering Project Management - A Novel Approach for Software Engineering Programs. Modern Applied Science, 5 (5), 87-104.

Caulfield, C., Xia, J., Veal, D., \& Maj, S. (2011). A Systematic Survey of Games Used for Software Engineering Education. Modern Applied Science , 5 (6), 28-43.

Kosa, M., Yilmaz, M., O'Connor, R., \& Clarke, P. (2016). Software Engineering Education and Games: A Systematic Literature Review. Journal of Universal Computer Science , 22 (12), 1558-1574.

Martí-Parreño, J., Méndez-Ibáñez, E., \& Alonso-Arroyo, A. (2016). The use of gamification in education: a bibliometric and text mining analysis. Journal of Computer Assisted Learning , 32 (6), 663-676.

Navarro, E. O., \& Hoek, A. v. (2004). SimSE: An Interactive Simulation Game for Software Engineering Education. Proceedings of the 7 th IASTED International Conference on Computers and Advanced Technology in Education. Kauai, Hawaii.

Pedreira, O., García, F., Brisaboa, N., \& Piattini, M. (2015). Gamification in software engineering - A systematic mapping. Information and Software Technology, 57, 157-168.

Petri, G., \& von Wangenheim, C. G. (2017). How games for computing education are evaluated? A systematic literature review. Computers \& Education , http://dx.doi.org/10.1016/j.compedu.2017.01.004 (In Press, Accepted Manuscript).

Sheth, S. K., Bell, J. S., \& Kaiser, G. E. (2012). Increasing Student Engagement in Software Engineering with Gamification. Columbia University Academic Commons.

Von Wangenheim, C. G., Savi, R., \& Borgatto, A. F. (2012). DELIVER! - An educational game for teaching Earned Value Management in computing courses. Information and Software Technology, 54 (3), 286-298.

Von Wangenheim, C. G., Thiry, M., \& Kochanski, D. (2009). Empirical evaluation of an educational game on software measurement. Empirical Software Engineering , 14 (4), 418-452.

Werbach, K., \& Hunter, D. (2012). For the win: How game thinking can revolutionize your business. Wharton Digital Press. 
Yildirim, I. (2017). The effects of gamification-based teaching practices on student achievement and students' attitudes toward lessons. The Internet and Higher Education , 33, 86-92.

Zyda, M. (2005). From visual simulation to virtual reality to games. Computer , 38 (September), 25-32. 